\title{
Predicting Pass Rates on the American Board of Internal Medicine Certifying Examination
}

\author{
Lisa K. Rollins, PhD, James R. Martindale, PhD, Michael Edmond, MD, Thomas Manser, MD, \\ W. Michael Scheld, MD
}

\begin{abstract}
Our objective was to determine the ability of the internal medicine In-Training Examination (ITE) to predict pass or fail outcomes on the American Board of Internal Medicine (ABIM) certifying examination and to develop an externally validated predictive model and a simple equation that can be used by residency directors to provide probability feedback for their residency programs. We collected a study sample of 155 internal medicine residents from the three Virginia internal medicine programs and a validation sample of 64 internal medicine residents from a residency program outside Virginia. Scores from both samples were collected across three class cohorts. The Kolmogorov-Smirnov $z$ test indicated no statistically significant difference between the distribution of scores for the two samples $(z=1.284, p=.074)$. Results of the logistic model yielded a statistically significant prediction of ABIM pass or fail performance from ITE scores (Wald = 35.49, $\mathrm{SE}=0.036, d f=1, p<.005)$ and overall correct classifications for the study sample and validation sample at $\mathbf{7 9} \%$ and $75 \%$, respectively. The ITE is a useful tool in assessing the likelihood of a resident's passing or failing the ABIM certifying examination but is less predictive for residents who received ITE scores between 49 and 66 .
\end{abstract}

KEY WORDS: certifying examination; in-training examination; education; predictions; residents. J GEN INTERN MED 1998;13:414-416.

$\mathbf{R}$ esidents in internal medicine must acquire competency in many content areas during their postgraduate training, including medical knowledge, clinical skills, clinical judgment, interpersonal skills, attitudes, ethics, and humanistic qualities as they apply across a vast range of content areas. ${ }^{1}$ To assist both residents and program directors, the American College of Physicians, the Association of Professors of Medicine, and the Association of Program Directors in Internal Medicine developed the first national In-Training Examination (ITE) in internal medicine in 1988. This examination was developed to serve as an assessment tool, primarily for residents at the midpoint of training during the second postgraduate year (PGY2). The ITE enables residents and program directors to receive feedback and identify potential deficiencies before administration of the American Board of Internal Medicine (ABIM) certifying examination.

Received from the University of Virginia School of Medicine, Charlottesville.

Address correspondence and reprint requests to Dr. Rollins: Box 401, HSC, University of Virginia School of Medicine, Charlottesville, VA 22908.
The ABIM certifying examination has been the source of a variety of studies over the last decade. Most of these studies dealt with the ability of the ABIM certifying examination to measure the construct of medical knowledge needed by an internal medicine physician. ${ }^{1-3}$ More recently, two studies have dealt specifically with the ability of the ITE to predict outcome on the ABIM certifying examination. ${ }^{4,5}$ Both studies demonstrated a significant correlation between the ITE and the ABIM certifying examination scores. However, neither study externally validated its results. Therefore, the purpose of this study was threefold: replicate the previous studies through an alternative mode of analysis (logistic model), develop a predictive model that is validated by external data, and develop a simple equation that can be utilized by residency directors in a spreadsheet format to provide quick and easy ABIM pass/fail probability feedback.

\section{METHODS}

Internal medicine residency directors from the three Virginia medical schools were asked to provide ITE scores obtained during residents' PGY2 year in 1992, 1993, and 1994, along with the ABIM pass/fail results for the corresponding classes in 1993, 1994, and 1995 who were taking the examination for the first time. The ITE scores reflected overall percentage correct as it appeared on the "Program Roster" report provided to residency directors. Any individuals from this cohort of residents who did not possess both scores or were administered the ITE during PGY1 or PGY3 were excluded from the study.

The validation data were acquired from an internal medicine residency program outside Virginia. The same criteria for inclusion into the data set were applied. The Kolmogorov-Smirnov two-sample $z$ test was conducted to assess whether the ITE scores from the validation sample were representative of the study sample.

To assess the ability of the ITE scores to predict pass/fail performance on the ABIM, a logistic regression model was used. The Wald statistic was used to test the null hypothesis that the calculated regression coefficient for ITE scores was 0. All data were analyzed using SPSS version 7 (Norusis MA, SPSS Advanced Statistics 6.1, SPSS Inc., Chicago, Ill.).

\section{RESULTS}

\section{Study Sample}

Included in the study were the ITE percentage correct and ABIM pass/fail scores from 155 residents. Of these, 
107 passed the ABIM certifying examination, while 48 failed, yielding an overall pass rate of $69 \%$. The mean (SD) on the ITE for the overall sample was $\bar{X}=64.45$ (10.20). The mean (SD) for those who passed the ABIM examination was $\bar{X}=68.59$ (8.68), and for those who failed, $\bar{X}=$ 55.21 (6.68).

\section{Validation Sample}

Included in the validation sample were ITE percentage correct and ABIM pass/fail scores from 64 internal medicine residents in a program outside Virginia. Within this sample, 42 passed the ABIM certifying examination while 22 failed, yielding an overall pass rate of $66 \%$. The ITE mean (SD) was $\bar{X}=61.53$ (8.23) for the overall sample. The mean (SD) for those who passed the ABIM examination was $\bar{X}=64.31$ (6.76), and for those who failed $\bar{X}=$ 56.23 (8.30).

\section{Analysis}

Results of the Kolmogorov-Smirnov $z$ test indicated no statistically significant difference between the distributions of ITE scores for the two samples $(z=1.284, p=.074)$.

Results of the logistic model as it was applied to the study sample indicated statistically significant prediction of ABIM pass/fail performance from ITE scores (Wald = 35.49, $\mathrm{SE}=0.036, d f=1, p<.005)$. In assessing the fit of the logistic model, $87 \%$ of the cases with passing performance on the ABIM examination were correctly classified, while $60 \%$ of the failing cases were correctly classified. The overall correct classification rate was $79 \%$. The prediction equation generated from the study sample was then applied to data from the validation sample. The resulting ability of the logistic model to predict ABIM pass/ fail outcome in the validation sample was similar to the result in the study sample, with an overall correct classification rate of $75 \%$. Table 1 shows the classification rates for both the study and validation samples.

\section{DISCUSSION}

Results from this study confirm findings from the two previous studies regarding the utility of the ITE in predicting the likelihood of a resident passing or failing the

Table 1. Fit of the Logistic Model to Sample and Validation Data

\begin{tabular}{lccccccc}
\hline \hline & \multicolumn{6}{c}{ Predicted } \\
\cline { 2 - 5 } & \multicolumn{3}{c}{ Study Sample } & & \multicolumn{2}{c}{ Validation Sample } \\
\cline { 2 - 4 } \cline { 5 - 7 } Observed & Pass & Fail & Correct, \% & Pass & Fail & Correct, \% \\
\hline Pass & 93 & 14 & 87 & & 37 & 5 & 88 \\
Fail & 19 & 29 & 60 & & 11 & 11 & 50 \\
$\begin{array}{l}\text { Overall correct } \\
\text { classification }\end{array}$ & & & 79 & & & 75 \\
\hline
\end{tabular}

ABIM. ${ }^{4,5}$ A logistic model was developed that yielded overall correct classifications for the study sample and validation sample at $79 \%$ and $75 \%$, respectively. However, it is clear that this model is better able to predict those persons who will pass the ABIM than those who will fail.

In addition, although the model was able to classify correctly at least three quarters of the overall cases, certain ITE scores were more highly predictive of passing or failing the ABIM examination. Specifically, ITE scores above 66 predicted a certain pass, while scores below 49 predicted certain failure. This compares with validation sample scores of 65 or above predicting a certain pass (excluding one outlying score of 74), and scores of 50 or below predicting certain failure.

This study also resulted in the development of a prediction equation that can enable residency directors to use residents' percentage correct ITE scores without having to convert the scores to percentiles. A spreadsheet can then be created that includes the resident's ITE percentage correct score in column $\mathrm{A}$, with the following formula used to convert the score to a probability of passing the ABIM certifying examination in column $\mathrm{B}$ :

$$
=1 /(1+(\operatorname{Power}(2.718,-(-12.4+0.2144 * \mathrm{~A} 1))))
$$

Utilization of this formula can enable residency directors to determine the probability of a resident passing or failing the ABIM examination, while taking into consideration the range of scores for which the model is less predictive.

Variation in samples must be considered when applying the prediction equation to a different cohort of residents. For example, smaller sample size and homogeneous samples will decrease the level of predictability of the model. However, the fact that the model effectively predicted outcome on the ABIM certifying examination across 3 years in a smaller validation sample indicates that the proposed model is able to maintain its utility across a different cohort of residents.

Evidence is fairly strong regarding the relation between the ITE and the ABIM examination. However, further research is needed to determine the most effective forms of remediation for those residents who score within the middle and lower ranges. In addition, many internal medicine residency programs currently encourage their residents to take ITE during both PGY1 and PGY2. However, it is unclear whether the administration of the ITE during PGY1 provides substantial benefit to residents in preparation for the ABIM examination. Nevertheless, evidence from this study supports the conclusion that the ITE is a useful tool in assessing the likelihood of a resident passing or failing the ABIM certifying examination.

\section{REFERENCES}

1. Norcini JJ, Grosso LJ, Shea JA, Webster GD. The relationship between features of residency training and ABIM certifying examination performance. J Gen Intern Med. 1987;2:330-6. 
2. Ramsey PG, Carline JD, Inui TS, Larson EB, LoGerfo JP, Wenrich MD. Predictive validity of certification by the American Board of Internal Medicine. Ann Intern Med. 1989;110:719-26.

3. Norcini JJ, Webster GD, Grosso LJ, Blank LL, Benson JA. Ratings of residents' clinical competence and performance on certification examination. J Med Educ. 1987;62:457-62.

4. Grossman RS, Fincher RE, Layne RD, Seelig CB, Berkowitz LR, Le- vine MA. Validity of the in-training examination for predicting American Board of Internal Medicine certifying examinatin scores. J Gen Intern Med. 1992;7:63-7.

5. Waxman H, Braunstein G, Dantzker D, et al. Performance on the internal medicine second-year residency in-training examination predicts the outcome of the ABIM certifying examination. J Gen Intern Med. 1994;9:692-4. 